\title{
Disorder-induced Fickian, yet non-Gaussian diffusion in heterogeneous media
}

\author{
Indrani Chakraborty ${ }^{1}{ }^{1}$ and Yael Roichman ${ }^{1,2, *}$ \\ ${ }^{1}$ School of Chemistry, Tel Aviv University, Tel Aviv 6997801, Israel \\ ${ }^{2}$ School of Physics and Astronomy, Tel Aviv University, Tel Aviv 6997801, Israel
}

(Received 24 September 2019; revised manuscript received 9 February 2020; accepted 31 March 2020; published 29 April 2020)

\begin{abstract}
Fickian yet non-Gaussian diffusion is observed in several biological and soft matter systems, yet the underlying mechanisms behind the emergence of non-Gaussianity while retaining a linear mean-square displacement remain speculative. Here, we characterize quantitatively the effect of spatial heterogeneities on the appearance of non-Gaussianity in Fickian diffusion. We study the diffusion of fluorescent colloidal particles in a matrix of micropillars having a range of structural configurations: from completely ordered to completely random. We show that non-Gaussianity emerges as a direct consequence of two coupled factors; individual particle diffusivities become spatially dependent in a heterogeneous randomly structured environment, and the spatial distribution of the particles varies significantly in such environments, further influencing the diffusivity of a single particle. The coupled mechanisms lead to a considerable non-Gaussian nature even due to weak disorder in the arrangement of the micropillars. A simple mathematical model validates our hypothesis that non-Gaussian yet Fickian diffusion in our system arises from the superstatistical behavior of the ensemble in a structurally heterogeneous environment. The two mechanisms identified here are relevant for many systems of crowded heterogeneous environments where non-Gaussian diffusion is frequently observed, for example, in biological systems, polymers, gels, and porous materials.
\end{abstract}

DOI: 10.1103/PhysRevResearch.2.022020

Einstein's theory of Brownian motion shows that for colloidal particles diffusing in two dimensions in a simple fluid, the mean-square displacement (MSD) is given by $\mathrm{MSD}=$ $4 D \tau$ where $D$ is the diffusion coefficient and $\tau$ is the lag time. The displacement distribution $G(\Delta x)$ for a Brownian particle is Gaussian. However, in many systems [1-13] such as granular materials [1], glassy materials [4], and biological systems [9-13], a non-Gaussian $G(\Delta x)$ has been observed. Usually, the non-Gaussian nature of $G(\Delta x)$ is related to a process of anomalous diffusion, with $\mathrm{MSD}=4 \tilde{D} \tau^{n}, n$ being the diffusion exponent. Surprisingly, in several cases diffusion has been observed to be Fickian but not Gaussian, that is, the MSD remains linear in time, but $G(\Delta x)$ is not Gaussian. This peculiar behavior has been referred to as "Anomalous, yet Brownian" or "Fickian yet non-Gaussian" diffusion (FNG), and has been observed in a wide variety of systems [10,1421] ranging from tracer colloids diffusing in suspensions of swimming microorganisms [14] to polymer chains diffusing on a surface $[17,18]$. Several theories have been put forward to explain such behavior which include the "diffusing diffusivity" model [22-25] that considers dynamic heterogeneities experienced by each colloidal particle in a changing environment. A second suggestion $[10,15,20,22]$ is that such motion

\footnotetext{
*roichman@tauex.tau.ac.il

Published by the American Physical Society under the terms of the Creative Commons Attribution 4.0 International license. Further distribution of this work must maintain attribution to the author(s) and the published article's title, journal citation, and DOI.
}

arises from the "superstatistical" behavior of an ensemble with each member having different diffusive parameters in a spatially varying environment. Guan et al. [20] observed FNG diffusion in a system of probe colloidal particles diffusing in a static matrix of densely packed bigger particles. It was postulated that the differences in the local configurations of the larger matrix particles led to the observed Fickian yet non-Gaussian diffusion. However, since the configuration of the matrix particles could not be controlled experimentally, it was not possible to quantitatively estimate the effect of the spatial heterogeneities on the non-Gaussian nature of the diffusion.

Here we study the emergence of non-Gaussianity from the structural randomness of the environment and quantify the percentage of disorder that gives rise to such non-Gaussian yet Fickian diffusion. This is achieved by fabricating arrays of micropillars with different degrees of randomness and tracking the motion of colloidal particles through them. We find that even a small degree of randomness leads to extensive non-Gaussianity due to two coupled mechanisms arising directly from the structural randomness in the micropillar arrays. Our experiments establish the great importance of structural disorder in FNG diffusion.

Our samples consist of micropillars (cylindrical cross section with diameter $=6 \mu \mathrm{m}$, height $=6 \mu \mathrm{m}$ ) made of the photoresist SU8 using standard photolithography techniques on glass coverslips. Samples with different area fractions and degrees of randomness were fabricated by designing corresponding masks (see Supplemental Material [26]). A flat glass control was made by exposing and developing an SU8 coated coverslip without introducing any mask. A suspension 

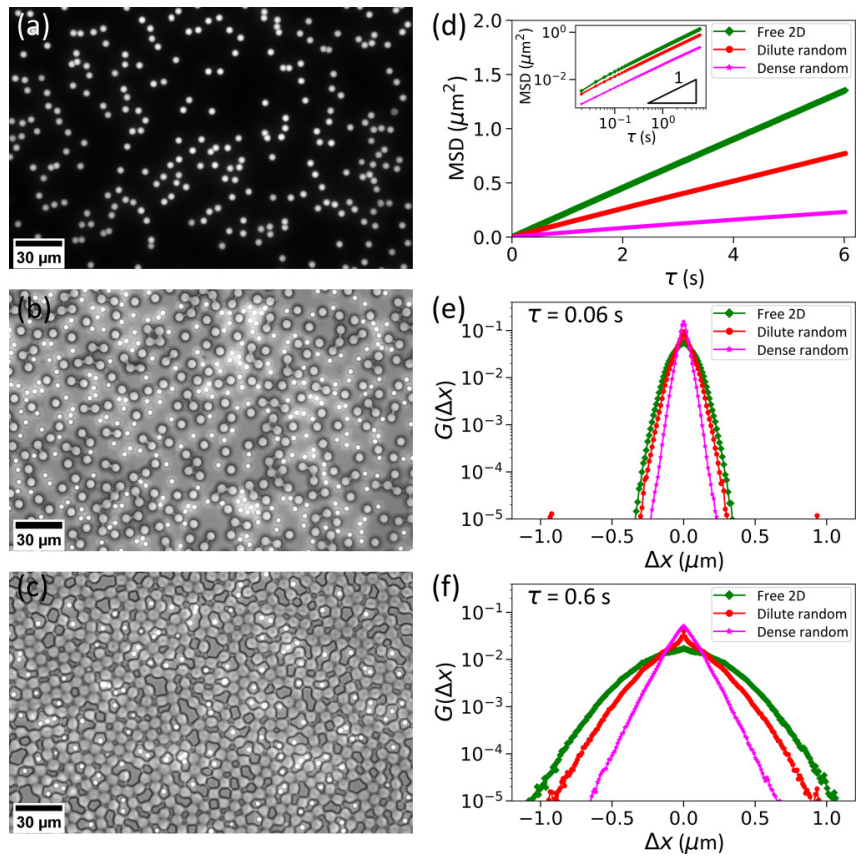

FIG. 1. Increase in density of the micropillars makes diffusion Fickian yet non-Gaussian. Microscope images in transmission mode of fluorescent tracer particles (bright spheres) diffusing on (a) a glass surface, i.e., "free 2D," (b) in a matrix containing an area fraction of $\phi=20 \%$ of randomly placed micropillars (bigger circles), i.e., "dilute random," and (c) in a matrix containing randomly placed micropillars at $\phi=72 \%$, i.e., "dense random." The time ensembleaveraged MSD plots are shown in (d) while the inset shows the log-log plots for the same systems indicating clear Fickian diffusion with $n=1$. $G(\Delta x)$ plots are shown for lag times (e) $\tau=0.06 \mathrm{~s}$ and (f) $\tau=0.6 \mathrm{~s}$. Note the distinct exponential behavior of the system with the dense random micropillars while the MSD is still clearly linear in time.

of fluorescent polystyrene particles $(4.19 \pm 0.27 \mu \mathrm{m}$ in diameter; Bangs Laboratories, Inc.) was placed onto the micropillar arrays and sealed with a top glass plate. Particles were allowed to sediment to the bottom of the chamber and diffuse there. The area fraction of the polystyrene particles on the glass surface was $\approx 6 \%$. The diffusion of the probe particles was imaged (Olympus IX-71 microscope, $40 \times$ objective) in both bright field and fluorescence modes and tracked at $50 \mathrm{fps}$ using conventional video microscopy $[27,28]$.

Initially we consider two cases of randomly arranged arrays of micropillars along with a control sample of polystyrene spheres diffusing in two dimensions on a planar glass surface [Fig. 1(a)]. In the first sample the micropillars have an area fraction of $\phi \approx 20 \%$ referred to as "dilute random" [Fig. 1(b)] while in the second case, the area fraction is $\phi \approx 72 \%$ referred to as "dense random" [Fig. 1(c)]. It should be noted that in the dense random case, the randomly placed micropillars were so close to each other that many of them were connected. This led to the formation of pockets separated by rigid walls in which particles could get trapped. The MSD of the particles in all three cases was observed to be linear, i.e., $n=1$, over a timescale spanning three decades [Fig. 1(d)]. In contrast, $G(\Delta x)$ is Gaussian only for the free
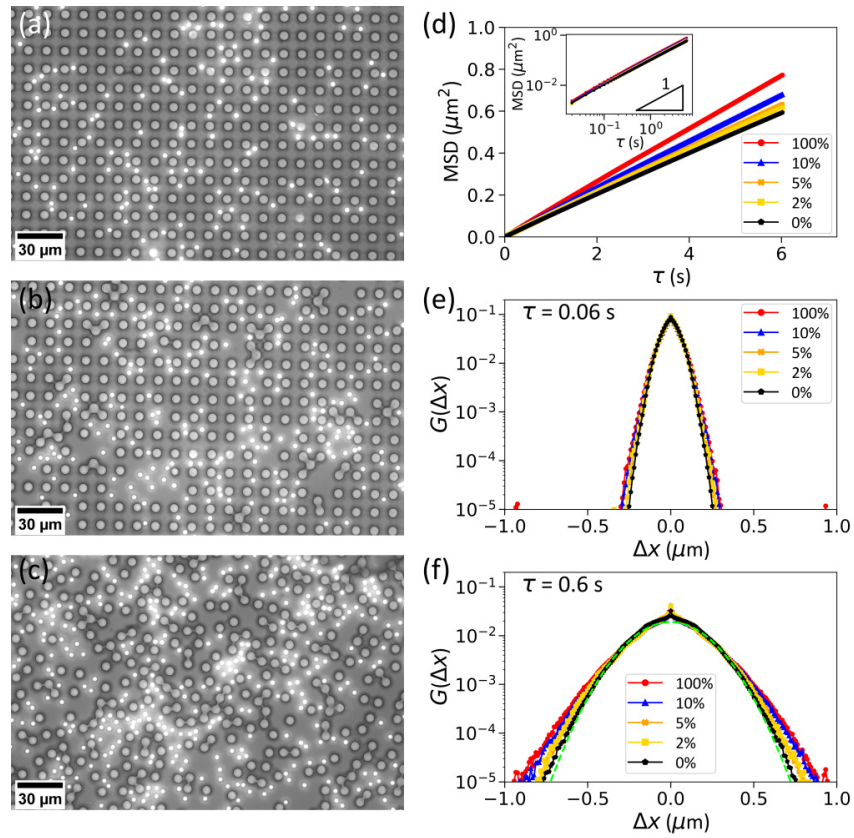

FIG. 2. Increase in randomness of the micropillars makes diffusion Fickian yet non-Gaussian. Fluorescent silica particles diffusing in a matrix of micropillars at $\phi=20 \%$ in (a) completely ordered (b) semirandom (10\% randomness) and (c) completely random (100\% randomness) arrangements. The MSD plots for randomness values of $0 \%, 2 \%, 5 \%, 10 \%$, and $100 \%$ are shown in (d) while the inset shows the log-log plot. $G(\Delta x)$ distribution is shown for lag times (e) $\tau=0.06 \mathrm{~s}$ and (f) $\tau=0.6 \mathrm{~s}$. The dashed green line in (f) shows a Gaussian fit to the $G(\Delta x)$ plot for the $100 \%$ random sample. Note the increasing non-Gaussian behavior with the increase of randomness even at the same area fraction.

diffusion samples and a markedly non-Gaussian distribution with a small peak centered at zero was obtained for all other samples [Figs. 1(e) and 1(f)]. For the dense random case, the change in $G(\Delta x)$ was even more dramatic having a clear exponential appearance. It should be noted here that at sufficiently long time intervals (lag time $>5 \mathrm{~s}$ ), the dense random sample showed $n=0.8$ instead of 1 , indicating a caging effect at these timescales. However, in the timescale in which the diffusion is Fickian, $G(\Delta x)$ clearly exhibits an exponential behavior. This indicates that an increase in area fraction of the micropillars leads to greater non-Gaussianity, in agreement with a previous report [29] which showed that increasing confinement leads to a larger non-Gaussianity in diffusion.

To explore the effect of the structural heterogeneity of the micropillars on the diffusion of the probe particles, we made a series of five samples each having $\phi=20 \%$ of micropillars (i.e., dilute random) with micropillar arrangements ranging from completely ordered to completely random. The samples were: (a) "ordered" with $0 \%$ randomness (square array with center-to-center distance of $12 \mu \mathrm{m}$ ) [Fig. 2(a)], "semirandom" including samples with (b) $2 \%$, (c) $5 \%$, and (d) $10 \%$ randomness [Fig. 2(b)], and (e) a $100 \%$ random sample [Fig. 2(c)]. The semirandom samples were obtained by choosing a given percentage of the micropillars randomly and then shifting them by a random amount from their positions 

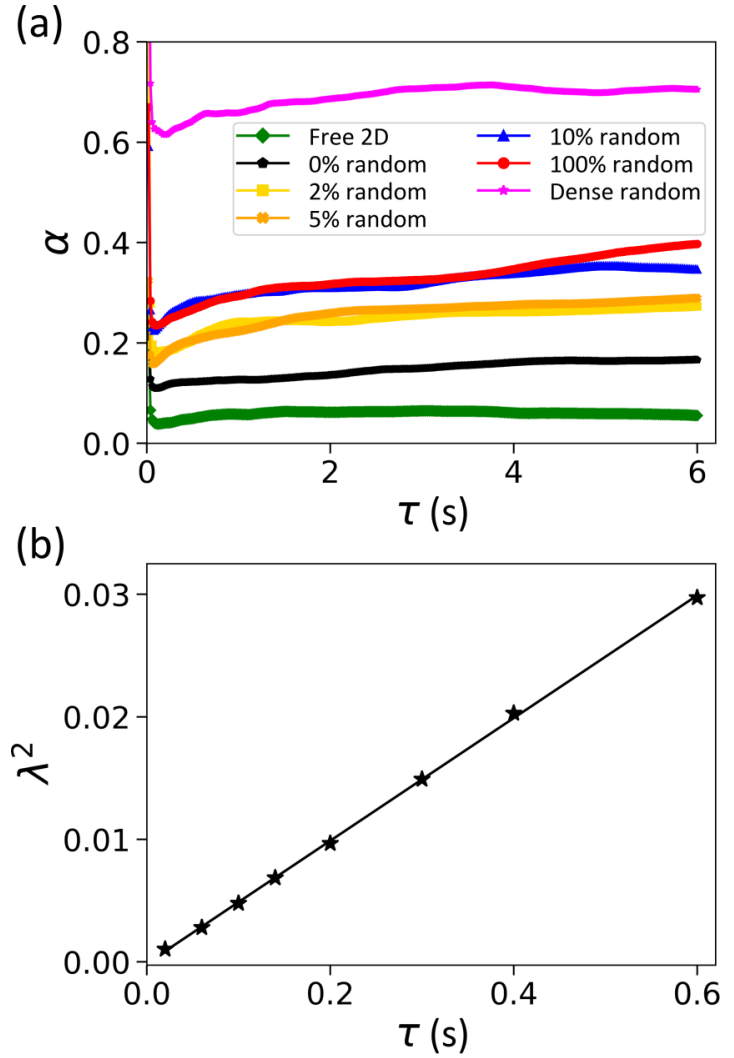

FIG. 3. Measurement of non-Gaussianity as a function of the randomness of the micropillars. (a) The non-Gaussian parameter for the seven different cases: free 2D, 0\%, 2\%, 5\%, 10\%, 100\% random, and dense random. (b) The value of the factor $\lambda^{2}$ obtained as a function of lag time is shown in (b). Note the excellent linear fit.

in the perfectly ordered structure while designing the mask. This is essentially equivalent to the introduction of a given percentage of "defects" in an ordered structure. All samples exhibited Fickian diffusion [Fig. 2(d)] with an increase in the diffusion constant as the structural randomness increased. However, $G(\Delta x)$ became less Gaussian as structural randomness increased [Figs. 2(e) and 2(f)]. We note that even with an inclusion of only $10 \%$ randomness in the structure, the $G(\Delta x)$ plots are very similar to the case for the $100 \%$ random structure, indicating very high sensitivity of the system to structural defects. This behavior is also reflected in the non-Gaussian parameter $\alpha$ [20], where $\alpha(t)=\frac{\left\langle\Delta x^{4}(t)\right\rangle}{3\left\langle\Delta x^{2}(t)\right\rangle^{2}}-1$ [Fig. 3(a)]. $\alpha$ is very close to zero for the free two-dimensional (2D) diffusion case, while it is $\approx 1.2$ times higher than that of the ordered micropillars for the $2 \%$ and $5 \%$ samples, and is nearly the same as for the $10 \%$ and $100 \%$ disordered samples. Notably, $\alpha$ remains nearly constant over several lag times for most cases. For the dense random case $\alpha$ is $\approx 5$ times that of the ordered case, indicating a very high degree of non-Gaussianity. We also verified the exponential nature of $G(\Delta x)$ for the dense random case [10] by fitting the equation $G(\Delta x)=A e^{-|x| / \lambda}$ to $G(\Delta x)$ plots over several different lag times and calculated the corresponding values of $\lambda$. From Fig. 3(b) we see that indeed $\lambda^{2} \sim \tau$, confirming the Fickian, yet exponential behavior. Note that emergence of non-Gaussianity with randomness is
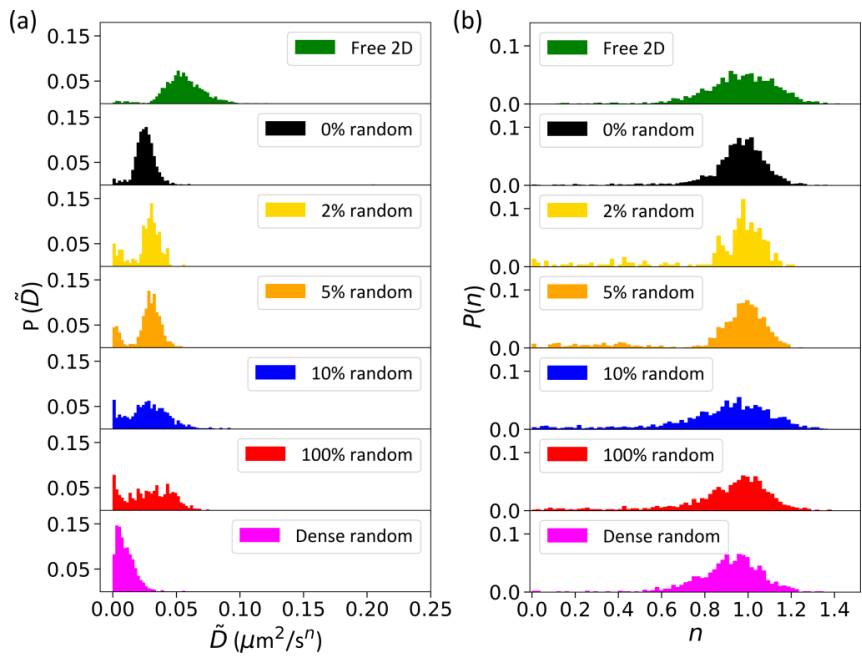

FIG. 4. (a) Probability distribution $P(\tilde{D})$ of the diffusion constant $(\tilde{D})$ for the seven cases: free $2 \mathrm{D}, 0 \%, 2 \%, 5 \%, 10 \%$ and $100 \%$ randomness, and dense random, respectively. Note the fingerprint of faster and slower populations in the $P(\tilde{D})$ plots for the dilute random samples with different degrees of randomness while the dense random sample shows a near-exponential behavior. (b) Probability distribution $P(n)$ of the diffusion exponents $(n)$ calculated from the linear fits of log-log MSD plots for each individual particle. Note that the distribution peaks about $n=1.0$, indicating predominantly normal diffusion for the majority of the particles for all the cases.

also observed at higher area fractions (28.3\%) of micropillars (see Supplemental Material Fig. S1 [26]).

To understand the mechanism behind the increased nonGaussianity due to density and structural randomness, we focus on the probability distribution of the diffusion constants $P(\tilde{D})$ over the ensemble for the seven systems: free $2 \mathrm{D}$, dilute random samples with randomness of $0 \%, 2 \%, 5 \%, 10 \%$, and $100 \%$, and the dense random case [Fig. 4(a)]. The diffusion constants here are calculated from the intercepts of the individual particle MSDs in log-log plots (see Supplemental Material [26]). For all the samples with different degrees of randomness $(2 \%-100 \%) P(\tilde{D})$ is not Gaussian, and has two notable features: (a) a second peak at lower values of the diffusion constant $(\tilde{D})$ appears, indicating that a portion of the ensemble moves very slowly, and (b) there is an extended tail at higher values of $\tilde{D}$ indicating that a portion of the ensemble moves faster than the average. For the dense random sample, the distribution looks almost exponential. In spite of such wide differences in the $P(\tilde{D})$ distributions, the single particle diffusion exponent distribution, $P(n)$, peaks at $n=$ 1.0 for all seven cases. This indicates Fickian diffusion on the single particle level for the great majority of the particles [Fig. 4(b)]. Further analysis shows that with an increasing degree of randomness, the small fraction of particles with $n \neq 1$ increases, along with an increase in the fraction of both slow and fast moving particles [see Supplemental Material Figs. S2(a) and S2(b) [26]]. This simultaneous increase in the fraction of very slow and very fast moving particles with increasing environmental disorder can only be attributed to the inherent structural heterogeneity of the system and is a 


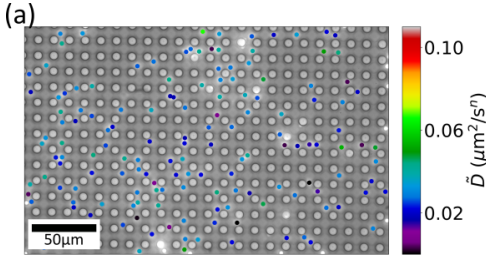

(b)
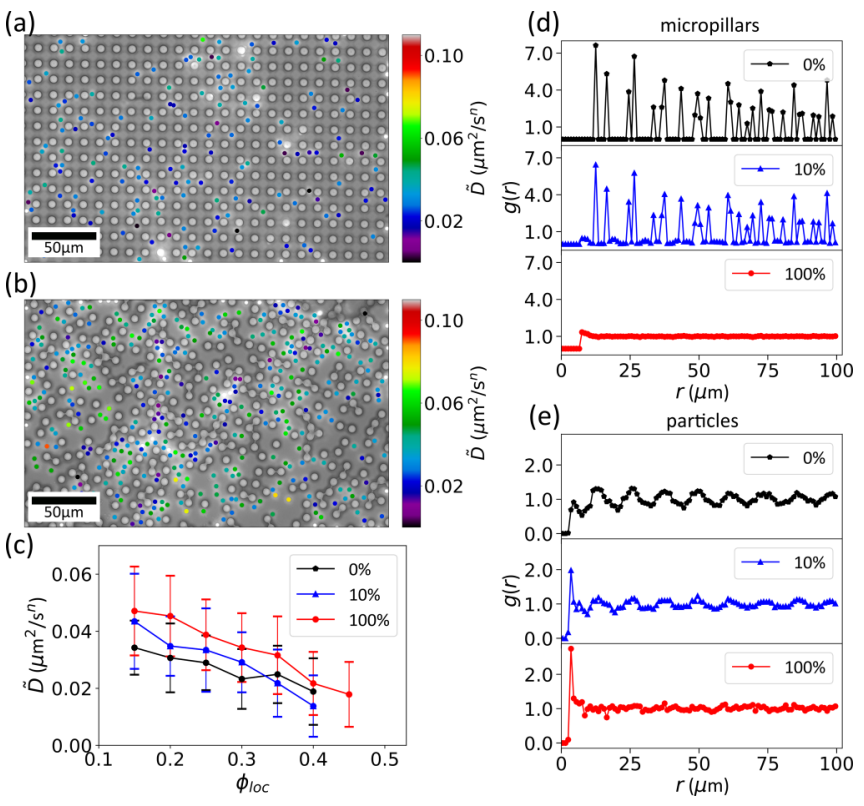

FIG. 5. 2D color maps representing the $\tilde{D}$ values of colloids for (a) $0 \%$ random and (b) $100 \%$ random micropillars. Note the higher $\tilde{D}$ values for particles in open spaces and lower $\tilde{D}$ values for particles close to a wall or other particles for the random sample. A plot showing the decrease of average $\tilde{D}$ with increasing local area fraction $\phi_{\text {loc }}$ (composed of micropillars and neighboring particles) around each given particle within a radius of interest of 50 pixels is shown in (c). Pair-correlation function $g(r)$ of the (d) micropillars and (e) particles for the $0 \%$ random, $10 \%$ random, and $100 \%$ random cases.

unique "fingerprint" of the effect of disorder on the diffusion of particles.

The explicit difference in $P(\tilde{D})$ for the random and ordered micropillars, therefore, points to the fact that the local spatial heterogeneities in the random structure result in the ensemble having subgroups of particles that see different local environments and consequently diffuse differently. In a random structure there are more open areas and narrow cavities as compared to an ordered structure. A particle can get trapped in the cavities surrounded by the micropillar walls on one hand, while on the other hand it might happen to be in a location which is far off from any micropillar wall. While the former leads to a slower motion or even caged diffusion, in the latter, the particle can diffuse unhindered. This can be seen in Figs. 5(a) and 5(b) where we show the $\tilde{D}$ values per particle for the $0 \%$ and $100 \%$ random samples in relation to their position in the matrix. An increase in the local area fraction $\phi_{\text {loc }}$ of neighboring features (both micropillars and particles) around each particle therefore leads to a lowering of the average $(\tilde{D})$ values as seen in Fig. 5(c). Another indication that the non-Gaussian distribution results from local density variations rather than the long-range order of the ordered sample is that the hydrodynamic interactions between distant tracer particles is similar for ordered and disordered samples at intermediate and long range (see Fig. S3 of the Supplemental Material [26]). The spatial distribution of the probe particles themselves is affected by structural randomness, and it varies more significantly in random samples. As a result, the diffusion of a particle entrapped in the crowded regions gets slowed down while diffusion is faster in regions with very few particles. Comparing the 2D pair-correlation function $g(r)$ for both the micropillars [Fig. 5(d)] and the particles [Fig. 5(e)] we see that for the ordered sample, $g(r)$, for the particles resembles that of the micropillars in the sense that both show periodic behaviors with peaks at 12,24 , and $36 \mu \mathrm{m}$, etc. In contrast, $g(r)$ for the $100 \%$ random case saturates very fast to 1.0 , indicating a wide distribution of the nearest- neighbor distances. Also the peak at $6 \mu \mathrm{m}$ is the highest for the particles in the random structure as compared to the very small peak in the ordered structure [Fig. 5(e)], indicating the presence of particles at very close distances to other particles or very crowded regions for the random arrangement. The amplified effect of the two coupled factors- the location dependence of the diffusivity values in a spatially heterogeneous structure, and slowing or speeding up of diffusion near crowded or free regionsproduces the extensive non-Gaussian response even at a dilute concentration of defects. The non-Gaussianity persists at both short and long timescales as seen from the nearly constant behavior of the non-Gaussian parameter over more than two decades of lag time (where the smallest time interval is $0.02 \mathrm{~s}$ ). This is because each individual particle does not move appreciably from its location within our experimental time frame, and does not experience the whole set of environments in the sample. In contrast, in the "diffusing diffusivity" model, each individual particle experiences a slowly fluctuating environment over their trajectory and the non-Gaussian behavior is seen for each particle. In that model, at longer timescales the diffusion of a single particle transitions to Gaussian behavior $[10,22]$.

To verify our hypothesis, we did a simple calculation. Assuming a Gaussian $G(\Delta x)$ for each particle, we took the weights of the different diffusion constant values from our measurements [Fig. 4(a)] and summed over the individual displacement probabilities using $G(\Delta x, t)=$ $\Sigma_{i=1}^{N} \frac{w_{i}}{\sqrt{4 \pi \tilde{D}_{i} t}} e^{-x^{2} / 4 \tilde{D}_{i} t}$ where $w_{i}$ is the weight of each given value of the diffusion constant $\tilde{D}_{i}$. Our calculation results at a lag time of $0.6 \mathrm{~s}$ [see Supplemental Material Figs. S4(a)-S4(g)] [26] agree well with our $G(\Delta x)$ measurements, indicating that indeed the spread in the diffusivities arising from the local spatial heterogeneities in a random structure is the reason behind the FNG diffusion in these systems. This is in essence superstatistical behavior where the fast, random motion of the colloidal particles is superposed with the variations in the environment with specific areas having higher and lower diffusivity values [22]. Previously, a diffusion constant distribution of the form $P(D)=\frac{1}{\langle D\rangle} e^{-D /\langle D\rangle}$ where $\langle D\rangle$ is the mean diffusion constant was shown to produce an exponential $G(\Delta x)[23,30]$. In our system, this is analogous to the dense random case.

In conclusion we experimentally showed that the existence of spatial heterogeneities is a profoundly important factor behind the emergence of non-Gaussianity in Fickian diffusion. Both the area fraction and structural randomness of the heterogeneities contribute directly to increasing the non-Gaussianity culminating in exponential displacement distributions in extreme cases. Even weak disorder in the system $(10 \%)$ can produce an extensive deviation from Gaussianity. 
This extreme sensitivity to randomness in the structure originates from the amplified contributions of two coupled effects; increased density variations in the matrix structure and in the tracer particle distribution. This combination leads to a wide range of diffusivities and produces the superstatistical nonGaussian behavior for Fickian diffusion. The importance of our study lies in the fact that it provides a fundamental insight into the role of structural heterogeneities in FNG diffusion. Our experiment separates the effects of temporal and spatial heterogeneities and provides clear evidence in support of the "superstatistics" argument in FNG diffusion. We expect this work to be an important addition in the endeavor to understand nonconventional diffusive behaviors in complex systems. Our findings are universally relevant in systems in which non-Gaussianity arises explicitly from the existence of spatiotemporal heterogeneities, for example, in diffusion of nutrients across the cell cytoplasm containing a sea of biomacromolecules [13], diffusion in protein crowded lipid bilayers [31], diffusion of dust particles in 2D equilibrium Yukawa liquids [32], liposomes diffusing in nematic solutions of F-actin filaments [15], colloidal particles diffusing among swimming cells [19] or in a dilute field of optical traps [33], and swimming microorganisms navigating through colloidal particles [34].

This research was supported by the Israel Science Foundation (Grant No. 988/17) and the PBC Postdoctoral fellowship of the Council for Higher education, Israel.
[1] A. V. Orpe and A. Kudrolli, Phys. Rev. Lett. 98, 238001 (2007).

[2] J. P. Gollub, J. Clarke, M. Gharib, B. Lane, and O. N. Mesquita, Phys. Rev. Lett. 67, 3507 (1991).

[3] O. J. N. Bertrand, D. K. Fygenson, and O. A. Saleh, Proc. Natl. Acad. Sci. USA 109, 17342 (2012).

[4] P. Chaudhuri, L. Berthier, and W. Kob, Phys. Rev. Lett. 99, 060604 (2007).

[5] M. J. Skaug, L. Wang, Y. Ding, and D. K. Schwartz, ACS Nano 9, 2148 (2015).

[6] D. Wang, H. Wu, L. Liu, J. Chen, and D. K. Schwartz, Phys. Rev. Lett. 123, 118002 (2019).

[7] T. Chen, H. J. Qian and Z. Y. Lu, Chem. Phys. Lett. 687, 96 (2017).

[8] A. A. Drăgulescu and V. M. Yakovenko, Quant. Financ. 2, 443 (2002).

[9] B. Stuhrmann, M. Soares e Silva, M. Depken, F. C. MacKintosh, and G. H. Koenderink, Phys. Rev. E 86, 020901(R) (2012).

[10] B. Wang, S. M. Anthony, S. C. Bae, and S. Granick, Proc. Natl. Acad. Sci. USA 106, 15160 (2009).

[11] W. He, H. Song, Y. Su, L. Geng, B. J. Ackerson, H. B. Peng, and P. Tong, Nat. Commun. 7, 11701 (2016).

[12] M. E. Grady, E. Parrish, M. A. Caporizzo, S. C. Seeger, R. J. Composto, and D. M. Eckmann, Soft Matter 13, 1873 (2017).

[13] P. Witzel, M. Götz, Y. Lanoiselée, T. Franosch, D. S. Grebenkov, and D. Heinrich, Biophys. J. 117, 203 (2019).

[14] K. C. Leptos, J. S. Guasto, J. P. Gollub, A. I. Pesci, and R. E. Goldstein, Phys. Rev. Lett. 103, 198103 (2009).

[15] B. Wang, J. Kuo, S. C. Bae, and S. Granick, Nat. Mater. 11, 481 (2012).

[16] I. Chakraborty, G. Rahamim, R. Avinery, Y. Roichman, and R. Beck, Nano Lett. 19, 6524 (2019).

[17] M. J. Skaug, J. Mabry, and D. K. Schwartz, Phys. Rev. Lett. 110, 256101 (2013).

[18] C. Yu, J. Guan, K. Chen, S. C. Bae, and S. Granick, ACS Nano 7, 9735 (2013).
[19] H. Kurtuldu, J. S. Guasto, K. A. Johnson, and J. P. Gollub, Proc. Natl. Acad. Sci. USA 108, 10391 (2011).

[20] J. Guan, B. Wang, and S. Granick, ACS Nano 8, 3331 (2014).

[21] A. L. Thorneywork, D. G. Aarts, J. Horbach, and R. P. Dullens, Soft Matter 12, 4129 (2016).

[22] A. V. Chechkin, F. Seno, R. Metzler, and I. M. Sokolov, Phys. Rev. X 7, 021002 (2017).

[23] M. V. Chubynsky and G. W. Slater, Phys. Rev. Lett. 113, 098302 (2014).

[24] Y. Lanoiselée and D. S. Grebenkov, J. Phys. A: Math. Theor. 51, 145602 (2018).

[25] P. Malgaretti, I. Pagonabarraga, and J. Rubi, Entropy 18, 394 (2016).

[26] See Supplemental Material at http://link.aps.org/supplemental/ 10.1103/PhysRevResearch.2.022020 for discussions on sample preparation and calculation of the diffusion constants, emergence of non-Gaussianity with randomness at a higher area fraction of micropillars $(28.3 \%)$, trend of variation in the fraction of particles which undergo subdiffusion, and slower and faster particles with randomness \%, two-point hydrodynamic correlation coefficients plot for the samples, and match between experimental and predicted $G(\Delta x)$ plots.

[27] D. Allan, T. Caswell, N. Keim, and C. van der Wel, Trackpy v0.3.0 Zenodo, 34028 (2015), https://zenodo.org/ record/34028\#.Xp7tMLm6bcs.

[28] J. C. Crocker and D. G. Grier, J. Colloid Interface Sci. 179, 298 (1996).

[29] K. He, S. T. Retterer, B. R. Srijanto, J. C. Conrad, and R. Krishnamoorti, ACS Nano 8, 4221 (2014).

[30] S. Hapca, J. W. Crawford, and I. M. Young, J. R. Soc., Interface 6, 111 (2009).

[31] J.-H. Jeon, M. Javanainen, H. Martinez-Seara, R. Metzler, and I. Vattulainen, Phys. Rev. X 6, 021006 (2016).

[32] Z. Ghannad, Phys. Rev. E 100, 033211 (2019).

[33] S. Mora and Y. Pomeau, Phys. Rev. E 98, 040101(R) (2018).

[34] R. Jeanneret, D. O. Pushkin, V. Kantsler, and M. Polin, Nat. Commun. 7, 12518 (2016). 